\title{
Organizational Robustness and Information Systems
}

\author{
Kevin C. Desouza \\ School of Management \& Centre for Future \\ Enterprise, QUT Business School, Queensland \\ University of Technology \\ kevin.desouza@qut.edu.au
}

\begin{abstract}
The concept of robustness is critically analyzed. Although robustness has been widely adopted across scientific disciplines, no comprehensive and systematic conceptualization on robustness exists. This paper develops a framework to conceptualize organizational robustness. The framework outlines mechanisms of organizational robustness in three layers - strategical mechanisms, functional mechanisms, and infrastructure mechanisms. The complex role of information systems for organizational robustness is also discussed.
\end{abstract}

Keywords: robustness; resilience; organization design; information systems;

\section{Introduction}

The robustness concept has been adopted in system biology [1], control theory [2], engineering [3], computer science [4], statistics [5], supply chain management [6], and many other scientific disciplines. Robustness examines how systems can remain stable in the face of uncertainties. In the management literature, robustness is predominantly examined in the fields of risk management and business continuity management [6]. Given the nature of volatility, uncertainty, and speed of disruption in today's environments, organizations are constantly challenged to maintain and bolster their robustness. It is thus critical to understand how organizations can maintain core functions against the uncertainties to achieve organizational robustness.

A critical perspective to study organizational robustness is the Information Systems (IS) perspective. With a broad perspective of IS, organizations can be understood as an IS and organizational robustness is essentially IS robustness. With a narrow perspective, organizational robustness can be critically affected by specific organizational IS (e.g. ERP) in a complex way. On one hand, IS can enable organizational robustness. For example, using information technologies can enhance organization's capabilities to sense signals of risks and mobilize responses in an agile and proactive manner. On the other hand, IS can also be a barrier to

\author{
Yancong Xie \\ Centre for Future Enterprise, QUT Business School, \\ Queensland University of Technology \\ yancong.xie@hdr.qut.edu.au
}

robustness. For instance, ERPs limit the ability of dynamic organizational change as they are quite cumbersome [7].

Robustness studies have a root in IS. Prior IS studies have already studied the relationship between IS and organizational robustness [8] [9]. Also, the discussions around robustness can potentially link well to emerging directions in IS [10] [11], such as augmented intelligence, IoT, blockchain, distributed work, worklife balance, and technostress.

Further, the significance to study organizational robustness has been greatly increased by recent COVID19. Given the COVID-19 ramifications and its disruptive impact on organizations (as well as all other forms of society), understandings of organizational robustness are critical, as it can provide insightful suggestions for organizations to survive global crises like COVID-19. There are also recent urgent calls to understand how IS can enable sustainability and organizational robustness [12] [13].

This paper clearly distinguishes robustness and resilience. Studying how IS affects organizational robustness, prior research has mainly examined resilience instead of robustness. Resilience has been conceptualized as a system quality to productively respond to uncertainties without engaging in an extended period of regressive behavior [8]. Although resilience and robustness can be intuitively similar, they have different emphasis [14]. Whereas resilience focuses on system capability to predict crisis and cope with crisis to return to a normal state (or a more desirable state), robustness emphasizes underlying system mechanisms to proactively and agilely respond to predictable and unpredictable crises. Generally, robustness is a broader concept than resilience, and resilience can reflect only one aspect of robustness. For example, in the context of cyber security, Desouza et al. [15] suggest that IS can be used by actors to undermine public institutions and disrupt political systems. In such a context, a resilient IS should be able to cope with incoming attacks, recover quickly from disruptions, and develop mechanisms or protocols to be immune to future similar attacks. In comparison, a robust system should be able to operate while under attacks by 
dynamically reconfiguring itself. A robust system should also be able to learn from the reconfiguration experiences and sharpen its capabilities in predicting, preparing, and shaping responses and actions for future various kinds of attacks.

Existing studies have extensively studied organizational resilience and have generated various valuable insights. However, armed with these findings, organizations still continue failing in recurrent crises. For example, the airline industry suffers every time with global crises (e.g. the oil crises, gulf war, SARS, economic crises, and COVID-19). It thus suggests that our understandings of organizational resilience are limited, which greatly motives this research to study organizational robustness.

Unfortunately, there is a dearth of studies on organizational robustness in the literature. Further, organizational robustness is examined only as one aspect of organizational resilience [6], suggesting an inaccurate conceptualization of robustness. To facilitate future studies on organizational robustness, this paper aims to systematically conceptualize robustness and embed the ideas into the organizational context, by integrating the extant literature across (a multitude of) several disciplines. An important issue to bear in mind: our focus is on developing a general-purpose framework for organizational robustness. In our approach, we take a broad view of the concept of organization. Organizations may be represented as a firm, an IS, or even an ensemble of human-machine mechanisms operating on a digital platform. To assist in an understanding to our approach, we will use economic organizations (firms) to guide the reader through the framework.

This paper is structured as follows. We firstly provide a definition of robustness in section 2, setting the scope and objectives of robustness studies. We then discuss the antecedents of robustness in section 3 . In section 4, we extend section 3 discussions in IS and provide comments about the effects of IS on organizational robustness. We conclude this paper in section 5 .

\section{Definition of robustness}

The robustness concept has been adopted in numerous fields in a multitude of ways. Table A1 in the appendix lists several definitions of robustness across different fields. Building on Kitano's work [1] in system biology, we define robustness as a property that allows a system to maintain its functions against perturbations in an environment. Four elements of robustness based on our definition are system, function, perturbation, and environment.

\subsection{System}

A system is a complex whole of interconnected individual parts [1]. A system is the unit of examination for robustness. Therefore, robustness is a system level property and it cannot be applied to the components which make up the system. Existing literature has two perspectives on the system element when examining robustness.

The first perspective involves viewing a system as an instrument [16]. An instrument refers to a model that incorporates a set of calculative processes to transform system inputs into system outputs. For example, adopting the system perspective of instrument, one may view organizations as business models [17]. Business model emphasizes a system-level, holistic approach to example how organizations "do business" [17]. For example, the business model of eBay is related to providing connections between individual suppliers and individual consumers using Internet technologies.

The second perspective views a system as a selforganizing autonomy [1]. A self-organizing autonomy refers to an entity that can operate without interventions and that can independently adjust its components. Unlike an instrument perspective that focuses on the nature of being used by third parties, self-organizing autonomy focuses on the self-regulation of the entity. While an instrument does not change too often, a selforganizing autonomy is typically dynamic. For example, when adopting the system perspective of selforganization autonomy, one may view organizations as comprising entities within a market. Each organization operates automatically in the market, aiming to maximize their objectives (e.g. performance) instead of seeking to globally optimize the overall market. In addition, organizations can independently change their structures based on their own utility maximization functions.

\subsection{Function}

Function of a system refers to a system trait, behavior, structure or output [18]. From the instrumental system perspective, function of a system is a property of system output. Here, the objective of robustness analysis is to make sure that it is reliable when an instrument is applied in different contexts. Therefore, a robust instrument is favored to generate correct outputs, regardless of the system input [5]. Correct system outputs can refer to the system output itself, such as the variance and average of the output numbers, or an implication regarding the system output, such as the validity of model predictions. If one aims to adopt the business model of eBay in their country (e.g. Alibaba), one must expect the same high profitability with their future business. In this example, the system function targeted to be stable is profitability. 
By contrast, from the self-organizing autonomy perspective, the function of a system is a property of system behavior or system structure. When examining self-organizing autonomies, robustness analysis focuses on emergent system properties, beyond simple system outputs. By emergent system properties, we refer to the system properties as the products of complex interactions of system components, rather than the products of individual system component [19]. For example, if viewing organizations as self-organizing autonomies, the functions of organizations can refer to organizational structure, for instance, administration hierarchy and production line hierarchy. Also, the functions of organizations can refer to organization's performance or organizational survival in a market.

\subsection{Perturbation}

Perturbation of a system refers to any deviation of a system's normal operations, that can affect the functions of a system. There are three main kinds of perturbations discussed in the literature [20] [21].

The first type of perturbation is system uncertainty. System uncertainty refers to the internal disruption of system structure. With any disruption of system structure, the configuration of system components can be changed. How systems generate outputs based on system inputs can be affected. Therefore, system functions can be influenced. One example of system uncertainty is organizational disruptions (e.g. logistical breakdowns). Organizational disruptions can change the configuration of organizational resources, which may further influence organization's operations [22].

The second type of perturbation is environmental disturbance. Environmental disturbance may refer to the variations of external factors that are related to system operations. Environmental disturbance may refer to variations in system inputs. Different system inputs can lead to different system functions, due to the imperfectness of system's calculative processes. For example, when organizations are viewed as instruments (e.g. business models), the input of organizations may be market settings. It is likely that business models can be effective in one market setting but not effective in others. Therefore, the variation of system inputs can affect system functions. Environmental disturbance can also be the variations of moderating factors over system operations. This type of environmental disturbance can change how systems generate outputs based on inputs, thus affecting system functions. For example, when viewing organizations as self-organizing autonomies, natural disasters such as floods and earthquakes can greatly affect organization's operations, thus affecting the organization's functions.

The third type of perturbation is called noise. Noise refers to the stochastic fluctuation within a system. The majority of real-world systems are non-deterministic [23]. They often involve random pathways to generate system outputs from system inputs. Any stochastic fluctuation in these random pathways may affect system functions. For instance, strategy-making in organizations can involve random processes [24]. Specifically, more recent literature has studied "shaping strategy" [25]. The term "shaping structure" reflects the "structuration" nature of strategy-making in organizations, in which top managers indirectly evolve strategy through their complex interactions with various stakeholders and organizational practices [26]. Therefore, the random processes, or noises, can affect organization's strategy-making, thus influencing organization's performance (i.e. system functions).

\subsection{Environment}

Environment of system refers to a context that specifies multiple heterogenous guidelines on system operations [13].

Environment can affect system functions, if the system functions are associated with the interactions between the focused system and other systems in the environment. One example system function mentioned above is organizational survival [22] [27]. Pajunen [22] notes that organizational survival is highly dependent on an organization's relationships with other organizations or actors. In other words, in different business environments, organizational survival can differ.

Environment can also affect system perturbation. Specifically, the scale of environmental disturbance is dependent on the environment. An example perturbation to organizations can be Internet disruption. In one country with poor telecom infrastructure, Internet disruptions can happen quite often; therefore, one single Internet disruption can be a small perturbation for an organization. However, in a country where Internet disruption is rare, one single Internet disruption can be significant. Survival amidst different scales of Internet disruption perturbations reflects on an organization's different degrees of robustness.

Most prior literature does not consider the environment element (see Table A1), possibly due to a presumption that environment is static. The notion of static environment suggests that the external environment settings of the system (as well as perturbations) remain unchanged in the process to achieve robustness. For example, when organizations cope with the disruptions of production lines, the technical and market demands of the organizations are assumed to remain the same. Given the nature of volatility and speed of technical revolution in today's environments, this simple assumption may be less accurate. It is unlikely that heterogenous guidelines specified by environment can ideally remain stable 
nowadays. For example, examining organizational robustness, the environment of an organization addresses market conditions, and political agendas that can shift dramatically over time. Additionally, environment is not an independent element and it can be affected by the process in which systems achieve robustness. For example, with the environmental disturbance on technology change, leading companies may come up with innovations intended to keep their performance. These innovations may later shift the way of business, thus re-shaping the business environment (e.g. digital imaging and digital printing replaced silver halide film and paper). Therefore, it is also critical to study robustness in static vs. dynamic environment.

\subsection{A typology of robustness}

We now provide a typology of robustness. This typology shows how these elements can be integrated to denote some general notions of robustness. The literature has examined two kinds of robustness, which we term as instrumental robustness and structural robustness. Advancing the existing understandings by introducing the dynamic environment element, we further propose a third type of robustness as cognitive robustness. Table 1 contains a summary of the three kinds of robustness.

Table 1. A summary of three kinds of robustness

\begin{tabular}{|l|l|l|l|}
\hline & $\begin{array}{l}\text { Instrumental } \\
\text { robustness }\end{array}$ & $\begin{array}{l}\text { Structural } \\
\text { robustness }\end{array}$ & $\begin{array}{l}\text { Cognitive } \\
\text { robustness }\end{array}$ \\
\hline $\begin{array}{l}\text { Objective of } \\
\text { robustness } \\
\text { analysis }\end{array}$ & $\begin{array}{l}\text { The external } \\
\text { validity of } \\
\text { instruments }\end{array}$ & $\begin{array}{l}\text { System stability } \\
\text { in a static } \\
\text { environment. }\end{array}$ & $\begin{array}{l}\text { System stability } \\
\text { in a dynamic } \\
\text { environment. }\end{array}$ \\
\hline $\begin{array}{l}\text { System } \\
\text { perspective }\end{array}$ & Instrument & $\begin{array}{l}\text { Self-organizing } \\
\text { autonomy }\end{array}$ & $\begin{array}{l}\text { Self-organizing } \\
\text { autonomy }\end{array}$ \\
\hline $\begin{array}{l}\text { Focused } \\
\text { perturbations }\end{array}$ & Noise & $\begin{array}{l}\text { System } \\
\text { uncertainty and } \\
\text { environmental } \\
\text { disturbance }\end{array}$ & $\begin{array}{l}\text { System } \\
\text { uncertainty, } \\
\text { environmental } \\
\text { disturbance, and } \\
\text { noise }\end{array}$ \\
\hline $\begin{array}{l}\text { Focused } \\
\text { functions }\end{array}$ & Properties of \\
system & $\begin{array}{l}\text { Properties of } \\
\text { system } \\
\text { behaviors or } \\
\text { system structure }\end{array}$ & $\begin{array}{l}\text { Properties of } \\
\text { system } \\
\text { behaviors or } \\
\text { system structure }\end{array}$ \\
\hline $\begin{array}{l}\text { Environment } \\
\text { assumption }\end{array}$ & Static & Static & Dynamic \\
\hline
\end{tabular}

The objective of instrumental robustness is to ensure that an instrument can be applied to different contexts [4]. The focused perturbation of this type of robustness is often noise [2]. Robustness analysis may be more critical in constructing an instrument, including more random paths. For example, many start-ups often imitate business models of existing, often famous entities (e.g. there are many contextualized instances of the sharing economy business model). The assumption is that these popular business models can work well in different business settings. Famous business models can imply this assumption, because such business models have been adopted in different contexts and have been tested to be robust. However, it is unsure whether less popular business models can be robust against various noises. Therefore, these less popular business models significantly need robustness analysis to be adopted by start-up founders.

Structural robustness focuses on ensuring that a selforganizing autonomy can maintain normal operations in terms of the core system functions [20]. Unlike instrumental robustness, the focused perturbations likely involve system uncertainty and environmental disturbance. Researchers and practitioners are interested in how organizations can automatically re-configure system components and change the system structure to remain stable on core functions. For example, stakeholders may use robustness analysis to analyze how well organizations are prepared for specific internal and external disruptions in a static environment.

Both instrumental robustness and structural robustness assume a static environment. Prior studies have not specifically studied robustness in a dynamic environment. In this paper, we term this type of robustness as cognitive robustness. Cognitive robustness investigates self-organization autonomies as well. However, the objective of robustness analysis is to ensure that a self-organizing autonomy can always maintain normal operations in a dynamic environment, i.e. how an organization demonstrates the highest performance during high market uncertainty. Focused perturbations include all three types of perturbations; system uncertainty, environmental disturbance, and noise. Noise may play a more significant role in this type of robustness than structural robustness because of the complex interplay between noise and the dynamics of environment. As noise can affect how systems move to a new state and adapt to the environment, it may also affect how environment is re-shaped by system adaptation process, which in turn may further determine future noises. To summarize, cognitive robustness is similar to structural robustness. However, cognitive robustness covers more kinds of perturbations and deals with the complex interactions between different kinds of perturbations as well as between perturbations and dynamic environment. While structural robustness talks about general system capabilities to achieve stability in ideal contexts, cognitive robustness focuses on the reflection of such capabilities in an agile manner in a dynamic environment.

\section{A layered view on robustness}

In the previous section, we conceptualize robustness. In this section, we discuss how robustness can be achieved with various mechanisms. Previous research has examined how systems can become robust [1] [28] [29]. Harmonizing existing knowledge, we 
provide a layered view of the mechanisms to achieve robustness as in Figure 1. The mapping of existing knowledge to our layered view is illustrated in Table A2.

\subsection{Top layer - strategical mechanisms}

Existing literature has identified two strategical mechanisms of robustness through persistence and adaptation [1]. Introducing a dynamic environment, we propose a third strategical mechanism of robustness as superposition.

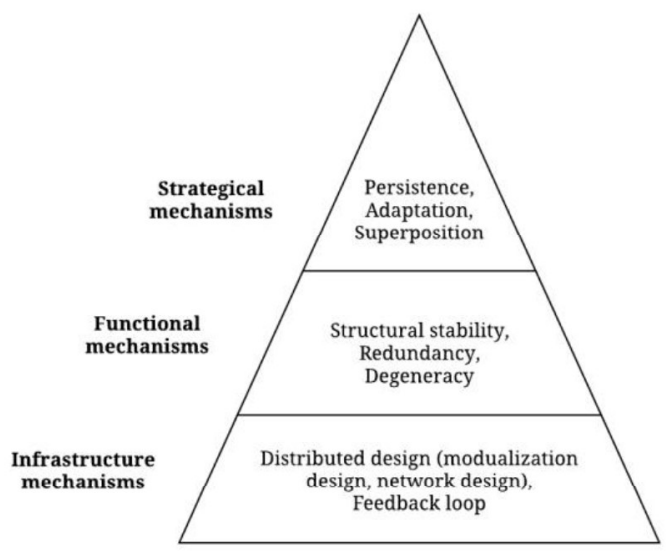

Figure 1. A layered view on robustness

Persistence suggests that systems can counter perturbations and maintain system functions without changing system operations [1] [30] [31]. As a result, perturbations can be "absorbed". Suppose an organization faces a blackout perturbation. If the organization can immediately switch to the backup powerline, its operations are hardly affected by the power disruption. In this case, the organization does not change its operations to survive the perturbation, thus adopting the persistence strategy.

The second reported strategical mechanism of robustness is adaptation [1] [30] [31]. Adaptation suggests that systems can reactively adjust to perturbations, and maintain system functions through arbitrarily changing system operations in a static environment. In the above example, suppose the organization does not have a backup powerline. The organization may use an outsourcing strategy to maintain its core functions of production. In this scenario, organizational robustness is achieved by revising organization's operations. Thus, the strategical mechanism is adaptation.

Beyond these two strategical mechanisms of robustness, both of which have received extensive coverage in the literature, there may be a third strategical mechanism of robustness, which we term as superposition. In quantum physics, the concept of superposition refers to the ability of a quantum system to be in multiple states at the same time until it is measured. We borrow this concept and refer to superposition, in the context of robustness, as a system ability to proactively adapt to perturbations, and maintain system functions through agilely changing system operations in a dynamic environment. Superposition differs from adaptation when considering the hyper turbulence of an environment. As environment can constantly change in a complex process, systems can continually adopt different states to maintain system functions. Given that a hyper turbulent environment can be difficult to predict, conversely the system state required to maintain the core functions become difficult to forecast as well. The state of a robust system can thus be highly uncertain, until it is observed after fixing the environment. Therefore, the state of a robust system can be in a superposition. Suppose an organization is constantly facing a series of unpredictable blackout disruptions in the above example. To achieve organizational robustness, the organization needs to act in an agile manner to adjust its outsourcing strategy to survive different degrees of blackout disruptions. Given that the outsourcing strategy may change frequently, the operations of the organization are highly uncertain; thus, the organization takes a superposition strategy to achieve organizational robustness.

To link the typology of robustness and strategical mechanisms of robustness, systems can adopt persistence strategy to achieve all three kinds of robustness. However, adaptation strategy is mostly adopted to achieve structural robustness while superposition strategy is mostly adopted to achieve cognitive robustness. Figure 2 illustrates the difference between the three strategical mechanisms of robustness.

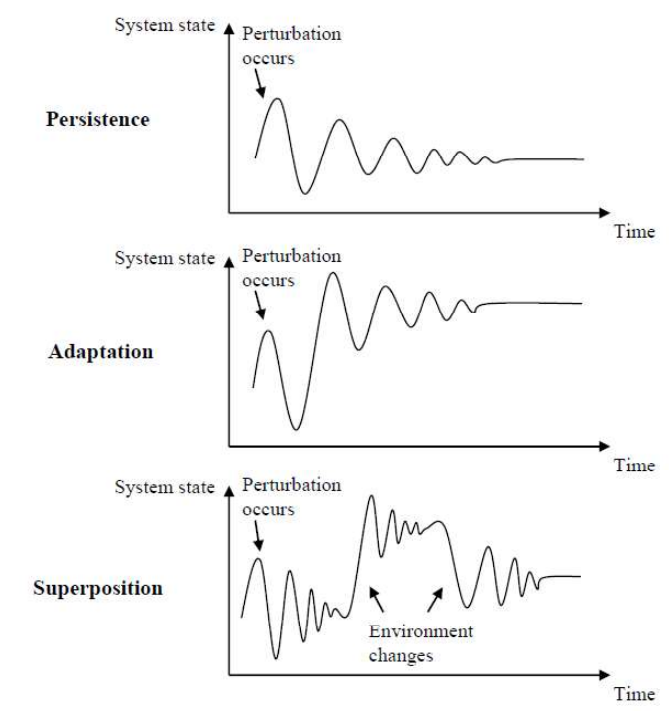

Figure 2. An example illustration of three different strategical mechanisms of robustness 


\subsection{Middle layer - functional mechanisms}

Strategical mechanisms at the top layer describe how robustness is achieved at a high level. In this section, we discuss how strategical mechanisms are enabled by functional mechanisms of robustness. The literature has reported many specific functional mechanisms, which can be mapped into three general types: structural stability [28], redundancy [1], and degeneracy [4].

Structural stability suggests that systems have a complex structural design that allows systems to be insensitive regarding system inputs. Therefore, the change of system input is unlikely to result in significant changes in system output, i.e. buffering [1], and system operations are thus less likely to be affected by perturbations. In other words, structural stability mainly enables persistence. One example of structural stability design is related to the collaborative environment in organizations. When organizations have multiple departments to cooperate on organizational projects, organizations can be less affected by the disruption of individual departments, thus being less sensitive to this type of system uncertainty.

Redundancy suggests that systems have redundant pathways in transforming system inputs into system outputs [32]. This is probably the most popular mechanism proposed in the existing literature [1] [4] [6] [18] [20] [28] [30] [33] [34] [35]. If a system has multiple same pathways from system inputs to system outputs, when one pathway is blocked due to perturbations, systems may immediately switch to alternative backup pathways. As a result, the normal system operation is not affected. Such systems demonstrate high persistence. One example of redundancy in organizational structure is when backup production lines are established. If current production lines experience disruptions, organizations can immediately switch to backup production lines. Thus, the production operation is not affected; organizations survive the disruption.

The last functional mechanism is called degeneracy [4]. In the biology discipline, degeneracy occurs when structurally dissimilar components, modules, or pathways can perform similar functions under certain conditions, but perform distinct functions in other conditions. Degeneracy suggests that systems have multiple heterogeneous pathways in transforming system inputs into system outputs. Although these pathways may be inherently different, they can still enable a viable connection from system inputs and system outputs [1] [30]. For example, organizations may have both data teams and non-data teams to provide consultancy services. When one type of team experience disruptions, organizations can use the other type of team to fulfill the business tasks.

\subsection{Bottom layer - infrastructure mechanisms}

The three kinds of functional mechanisms can be enabled by two major types of infrastructure mechanisms of robustness - distributed design and feedback loop design [1].

Distributed design includes modularization design and network design. Modularization means disintegrating system resources, tasks, and autonomy into functionally sperate components, i.e. modules [1]. Network design, or architectural framework, is a hierarchical design of connecting individual modules [1]. Together, modularization design and network design bring complexity to system structure, which allows systems to operate in nuanced processes. For example, most organizations have separate departments that have different business responsibilities; each department is further split into several independent teams, reflecting the modulation design. Further, in one organization, departments and teams have complex interplays. For instance, the delivery of organizational projects may need the collaboration of multiple teams from different departments; for different organizational projects, the collaboration pattern can be completely different. Such collaborative connections then reflect the networking design of connecting modules.

Distributed design can enable structural stability by introducing complexity to the system design [1] [33]. Without distributed design, how systems transform system inputs into system outputs is mostly linear. A change of system inputs is very likely to change system outputs in a single direction. Therefore, systems tend to be sensitive to system inputs. However, with a distributed design, system operations can become complex and can become increasingly non-linear. The pathways from system inputs to system outputs will become unpredictable. The change of system inputs is less likely to systematically impact system outputs; structural stability is ensured. For example, when organizations rely on various information sources to generate business analysis reports, the disruption of one information source can hardly affect generated reports.

Redundancy can also be easily realized with distributed design, if systems have backup modules. These backup modules are the same as the original modules. When the original modules are damaged, backup modules can immediately replace the original modules, enabling the same pathway from system inputs and system outputs. Therefore, redundancy is achieved.

Distributed design can also ensure degeneracy by introducing complexity. As the distributed design can connect system modules in a complex way, there may be diverse heterogeneous pathways transforming system inputs into system outputs. Hence, degeneracy is ensured. For example, the distributed digital platform design can provide effective mechanisms to 
organizational innovations, thus enhancing organization's degeneracy design.

Another core infrastructure mechanism is feedback loop design. Feedback loop is a development control circuit that enables a system to change its distributed design, based on past operations [1] [36].

Feedback loop can enable distributed design by revising the modularization design. This is done through so-called decoupling and coupling [1]. Decoupling suggests decomposing existing modules into granular resources and coupling suggests the opposite. This approach can enhance redundancy, if decoupling and coupling generate modules identical to existing ones, and degeneracy, if decoupling and coupling generate innovative modules from existing ones.

Feedback loop can also influence distributed design through network design [1]. Revising network design means revising the protocols in guiding the hierarchy of modules. With this approach, feedback loop can especially contribute to degeneracy, since revising the connections of modules is highly likely to enable new pathways from system inputs to system outputs.

\section{The complex role of IS for robustness}

At the bottom layer, the use of IS can enhance distributed design. On one hand, the implementation of IS reflects organization's practice of modulization design, as individual IS is designed to incorporate relevant resources for specialized tasks (e.g. CRM and ERP). Thus, IS are essentially designed as modules. Also, the use of IS can promote the storage, integration, and management of organizational resources, thus enhancing the modulization design. For example, the literature has suggested that digital platforms such as online marketplaces (e.g. Amazon) or software as a service can realize the distributed design, thus promoting modular/component development and facilitating innovations [37]. On the other hand, the use of IS can greatly facilitate communications by providing new effective communication mediums and reducing communication costs. For example, using IS, organizations may adopt standardized modeling languages (e.g. BPMN and UML), which can provide intuitive and systematic protocols for stakeholders from different backgrounds to effectively communicate with each other. Therefore, the information flows within organizations and network design can be enhanced.

At the bottom layer, the use of IS can also enhance feedback loop design. The use of IS can enhance organization's capability to sense signals, as IS can enable faster information gathering and transmission. For example, social media has been exponentially adopted by organizations in external stakeholder engagement, which may take a long time to accomplish with conventional methods such as surveys. In the context of Internet of Things, organizations adopt smart devices (e.g. sensors and edge computing platforms) in their IS. These devices can enable real-time sensing of environmental information and agile data analytics to enhance organizational capability of information perception [32]. Also, the use of IS can enhance the organizational capability to make quicker and wiser responses based on perceived signals. As commented earlier, the use of IS can greatly facilitate communications in organizations. Executives can thus make decisions and deploy decisions shortly. For example, conventionally, lots of paper works need to be done before executives finally approve change proposals. With IS deployed and business processes digitalized, the change proposals can be approved and deployed in a much faster manner. In addition, IS can undertake data analytics and can promote organizational knowledge, with which executives can make better decisions responding to perceived signals. For instance, there is a recent growing interest in utilizing big data analysis in IS, which can greatly promote knowledge generation and decision making [38]. Further, IS can also facilitate collaborations and enable collective wisdom in decision-making. One example is adopting sharing economy platforms such as Wikipedia, where individual users can collaborate to generate wisdom.

More intuitively, at the middle layer, the positive effects of IS on organizational robustness can be reflected in the three functional mechanisms of robustness. IS can promote structural stability as ISs are well-structured components of organizations. IS itself has the capacity to absorb errors, thus keeping organizational operations unaffected. For example, IS (e.g. ERPs) can check manual errors and reduce the negative impacts of these errors. Also, the use of IS can help organizations to quickly identify and address risks, before tremendous negative impacts are created. For instance, Nan and $\mathrm{Lu}$ [39] find that organizations can use information from digital platforms to effectively manage an earthquake-induced organizational crisis.

IS can increase redundancy as it can offer alternatives for organizational operations. First, the design of IS has embraced the redundancy design. Multiple mechanisms have been implemented to ensure that the IS can work properly during disruptions. For example, databases in most IS normally use backup servers to store data at the physical layer, which is an ideal reflection of the redundancy design. Second, IS are digital alternatives to conventional processes of organizational operation. Even when the entire IS breaks down, organizations can still turn to conventional manual approaches. Therefore, the implementation of IS can enhance the redundancy design.

Further, IS can also facilitate degeneracy. IS can effectively store, manage, and integrate organizational 
resources, thus enabling innovative utilization of organizational resources. This effect aligns very well with the IS innovation research [40] [41]. According to Swanson's tri-core model, IS innovations can be categorized into three types 1 ) innovations related to IS tasks, 2) innovations related to administration of the business, and 3) innovations related to the core technology of business [40]. It thus suggests that the use of IS can enhance degeneracy at the three dimensions.

Positively affecting the antecedents of robustness, IS can have positive effects of on organizational robustness. Thus, we propose:

Proposition 1: The use of IS can promote organizational robustness.

Despite the positive effects on organizational robustness, IS can also be a barrier to organizational robustness. This can be related to the heated discussion in the robustness literature regarding the relationship between robustness and fragility. Intuitively, when systems are more robust, they can be considered having superior system designs and being less fragile. Yet, the literature has suggested otherwise [1]. When system robustness increases, system fragility can also increase. Given that perturbations can be distinct in nature, when systems are highly robust towards some perturbations, systems can be highly fragile to other perturbations [33]. For example, in order to be robust against the increase of online demands, organizations may implement a higher degree of digitalization (e.g. configure more IT resources). As a result, organizations can be more fragile, i.e. less robust, towards a blackout perturbation.

In the IS context, the use of IS can hinder organizational robustness for hindering organizational agility. Organization agility refers to "a set of processes that allows an organization to sense changes in the internal and external environment, respond efficiently and effectively in a timely and cost-effective manner, and learn from the experience to improve the competencies of the organization" [42]. Organizational agility is essential for organization's cognitive robustness in the current turbulent business environment, as organizations need to agilely adapt to the fast-changing perturbations to stay robust. Seo and La Paz [42] has listed many reasons for the negative effects of IS on organizational agility, such as the overwhelming collection of data and the inflexibility of IS. Building on this knowledge, we propose two major causes to explain the negative influence of IS on organizational robustness.

First, the development of IS can lead to errors, which prevents organizations from adapting to perturbations wisely. Currently, most organizations take the agile project development approach to develop IS, which focuses on module add-ons. This approach has limitations regarding the integration of IS modules, resulting in IS disruptions. The negative effect can be more significant with a larger IS. When organizations adopt larger IS, IS may create more errors (e.g. inconsistency of data format between different modules), thus negatively affecting organization coping with perturbations. For example, the tragedy of Boeing 737 max crashes was majorly due to the integration of computer system modules. These modules individually work fine, but the collective system can have fatal errors in critical situations. Other famous examples include Nike's i2 failure in 2000 and General Motor's faulty ignition switch in 2014.

Second, large IS increases inflexibility and organization's unwillingness to change. When organizations implement cumbersome IS, organizations tend to be more reluctant to adopt dramatic changes to their IS and adopt new means of operations. Organizations may be delayed to cope with new types of perturbations. Further, many ISs encode processes and rules that govern organization operations. As such, even if the organization wants to change direction/focus, it might be limited to how quickly they can get the IS to change to achieve robustness [7].

The negative effect of IS is more salient nowadays. Today's business environment is more turbulent, characterized by more surprising, inconsistent, unpredictable, uncertain, and frequent perturbations [43]. To survive in such an environment, organizations need to take the superposition strategy to achieve cognitive robustness. Organizations need to be able to repurpose, innovate, and re-orient, components (resources, processes, capabilities, and assets) of the organization in novel ways in an agile manner. In such a context, the dark sides of IS on organizational robustness may result in more significant negative consequences.

To summarize the negative effect of IS on organizational robustness, we propose:

Proposition 2: The use of IS can negatively affect organizational robustness.

\section{Conclusions}

In this paper, we provide a framework for understanding robustness in the context of organization and information systems. We provide a comprehensive definition of robustness including a detailed discussion of different elements of robustness. We then present a novel layered view on mechanisms used to achieve robustness. Further, we initialize the discussions around the complex role of IS for organizational robustness.

Our major contributions are twofold. First, we blend the existing understanding of robustness in different fields and provide foundational discussions for future studies examining robustness. We contribute to the terminology of robustness and facilitate future 
communications on this important concept. We are the first, to our knowledge, to discuss the relationship between IS and organizational robustness. Second, we develop the current knowledge of robustness by introducing the dynamic environment element. From this novel perspective, we propose a new type of robustness (i.e. cognitive robustness) and a new strategical mechanism of robustness (i.e. superposition strategy), initializing a novel promising research stream to study robustness.

\section{References}

[1] Kitano, H., "Biological Robustness", Nature Reviews Genetics (5:11), 2004, pp.826-837.

[2] Alippi, C., "Robustness Analysis", In Intelligence for Embedded Systems, pp. 95-12, Springer, Cham, 2014

[3] Fuchs, M., Girimonte, D., Izzo, D. and Neumaier, A., "Robust and Automated Space System Design", In Robust Intelligent Systems, pp. 251-271. Springer, London, 2008.

[4] Sussman, G.J., "Building Robust Systems an Essay", Citeseer (113), 2007, p.1324.

[5] Bradley, J.V., "Robustness?", British Journal of Mathematical and Statistical Psychology (31:2), 1978, pp.144-152.

[6] Durach, C. F., Wieland, A., and Machuca, J. A. D., "Antecedents and Dimensions of Supply Chain Robustness: a Systematic Literature Review". International Journal of Physical Distribution \& Logistics Management (45:1/2), 2015, pp 118-137.

[7] Desouza, K., 2006. Agile IS. Routledge.

[8] Riolli, L. and Savicki, V., "Information System Organizational Resilience", Omega (31:3), 2003, pp.227-233.

[9] Wang, J.W., Gao, F. and Ip, W.H., "Measurement of Resilience and Its Application to Enterprise IS", Enterprise IS (4:2), 2010, pp. $215-223$.

[10] Forman, C., King, J.L. and Lyytinen, K., "Special Section Introduction-Information, Technology, and the Changing Nature of Work", IS Research (25:4), 2014, pp.789-795.

[11] Jain, H., Padmanabhan, B., Pavlou, P.A. and Santanam, R.T. eds., "Call for Papers-Special Issue of IS Research-Humans, Algorithms, and Augmented Intelligence: The Future of Work, Organizations, and Society", IS Research (29:1), 2018, pp.250-251.

[12] Rai, A., "The COVID-19 Pandemic: Building Resilience with IS Research", MIS Quarterly (44:2), 2020, pp.iii-vii.

[13] Sakurai, M. and Chughtai, H., "Resilience Against Crises: COVID-19 and Lessons From Natural Disasters", European Journal of IS, 2020, pp.1-10.

[14] Maurer, F. and Schumacher, J., "Organizational Robustness and Resilience as Catalyst to Boost Innovation in Smart Service Factories of the Future", In 2018 IEEE International Conference on Engineering, Technology and Innovation (ICE/ITMC), 2018, pp. 1-9. [15] Desouza, K.C., Ahmad, A., Naseer, H. and Sharma, M., "Weaponizing IS for political disruption: The Actor, Lever, Effects, and Response Taxonomy (ALERT)", Computers \& Security (88), 2020, p.1-15.

[16] Xu, H. and Mannor, S., "Robustness and Generalization", Machine Learning (86:3), 2012, pp.391-423.

[17] Zott, C., Amit, R. and Massa, L., "The Business Model: Recent Developments and Future Research", Journal of Management (37:4), 2011, pp.1019-104.

[18] Félix, M.A. and Barkoulas, M., "Pervasive Robustness in Biological Systems", Nature Reviews Genetics (16:8), 2015, pp.483496.

[19] Holland, J.H., Emergence: From Chaos to Order, OUP Oxford, 2000.

[20] Fares, M.A., "The Origins of Mutational Robustness", Trends in Genetics (31:7), 2015, pp.373-381.
[21] Wagner, A., "Robustness, Evolvability, and Neutrality", FEBS letters (579:8), 2005, pp.1772-1778.

[22] Pajunen, K., "Stakeholder Influences in Organizational Survival", Journal of Management Studies (43:6), 2006, pp.12611288.

[23] Holland, J.H., Hidden Order: How Adaptation Builds Complexity, Cambridge, Massachusetts, 1995.

[24] Cohen, M.D., March, J.G. and Olsen, J.P., "A Garbage Can Model of Organizational Choice", Administrative science quarterly, 1972, pp.1-25.

[25] Pettigrew, A.M. "Longitudinal Field Research on Change: Theory and Practice", Organization Science (1:3), 1990, pp. 267-292. [26] Jarzabkowski, P., "Shaping Strategy as a Structuration Process", Academy of Management Journal (51:4), 2008, pp.621-650

[27] Lamberg, J.A., Tikkanen, H., Nokelainen, T. and Suur Inkeroinen, H., "Competitive Dynamics, Strategic Consistency, and Organizational Survival”, Strategic Management Journal (30:1), 2009, pp.45-60.

[28] Krakauer, D.C., "Robustness in Biological Systems: a Provisional Taxonomy". In Complex Systems Science in Biomedicine, pp. 183-205, Springer, Boston, MA, 2006.

[29] Wagner, A., Robustness and Evolvability in Living Systems. Princeton University Press, 2013.

[30] Félix, M.A. and Wagner, A., "Robustness and Evolution: Concepts, Insights and Challenges from a Developmental Model System", Heredity (100:2), 2008, pp.132-140.

[31] Kitano, H., "Towards a Theory of Biological Robustness", Molecular Systems Biology (3:1), 2007, pp.1-7.

[32] Henry, M., Leach, F., Davy, M., Bushuev, O., Tombs, M., Zhou, F. and Karout, S., "The Prism: Efficient Signal Processing for the Internet of Things", IEEE Industrial Electronics Magazine (11:4), 2017, pp.22-32.

[33] Carlson, J.M. and Doyle, J., "Complexity and Robustness", Proceedings of the National Academy of Sciences (99: suppl 1), 2002, pp. 2538-2545

[34] Masel, J. and Trotter, M.V., "Robustness and Evolvability", Trends in Genetics (26:9), 2010, pp.406-414.

[35] Wagner, A., "Distributed Robustness Versus Redundancy as Causes of Mutational Robustness", Bioessays (27:2), 2005, pp.176188.

[36] Stelling, J., Sauer, U., Szallasi, Z., Doyle III, F.J. and Doyle, J., "Robustness of Cellular Functions", Cell (118:6), 2004, pp.675-685.

[37] Tiwana, A., "Evolutionary Competition in Platform Ecosystems", IS Research (26:2), 2015, pp.266-281.

[38] Abbasi, A., Sarker, S. and Chiang, R.H., "Big Data Research in IS: Toward an Inclusive Research Agenda", Journal of the Association for Information Systems (17:2), 2016, p. i-xxxii.

[39] Nan, N. and Lu, Y., "Harnessing the Power of Self-organization in an Online Community During Organizational Crisis", MIS Quarterly (38:4), 2014, pp.1135-1158.

[40] Swanson, E.B., "IS Innovation Among Organizations", Management Science (40:9), 1994, pp.1069-1092

[41] Swanson, E.B. and Ramiller, N.C., "The Organizing Vision in IS Innovation". Organization Science (8:5), 1997, pp.458-474.

[42] Seo, D. and La Paz, A.I., "Exploring the Dark Side of IS in Achieving Organizational Agility", Communications of the ACM (51:11), 2008, pp.136-139.

[43] Ansell, C., Sørensen, E. and Torfing, J., "The COVID-19 Pandemic as a Game Changer for Public Administration and Leadership? The Need for Robust Governance Responses to Turbulent Problems", Public Management Review, 2020, pp.1-12

[44] Levin, S.A. and Lubchenco, J., "Resilience, Robustness, and Marine Ecosystem-based Management", Bioscience (58:1), 2008, pp.27-32.

[45] Li, Y., Kim, Y.M., Mintarno, E., Gardner, D.S. and Mitra, S., "Overcoming Early-life failure and Aging for Robust Systems", IEEE Design \& Test of Computers (26:6), 2009. pp.28-39.

[46] Woodward, J., "Some Varieties of Robustness", Journal of Economic Methodology (13:2), 2006, pp.219-240. 


\section{Appendix}

\begin{tabular}{|c|c|c|}
\hline Discipline & Definition of robustness & Source \\
\hline \multirow{6}{*}{$\begin{array}{l}\text { System } \\
\text { biology }\end{array}$} & Robustness is the persistence of an organismal trait under perturbations. & [30] \\
\hline & $\begin{array}{l}\text { Robustness is a property that allows a system to maintain its functions against internal and external } \\
\text { perturbations. }\end{array}$ & [1] \\
\hline & $\begin{array}{l}\text { Robustness, the ability to maintain performance in the face of perturbations and uncertainty, is a long- } \\
\text { recognized key property of living systems. }\end{array}$ & [36] \\
\hline & $\begin{array}{l}\text { Robustness can be defined and measured as the average effect of a specified perturbation on a specified } \\
\text { phenotype. }\end{array}$ & [34] \\
\hline & A biological system is robust to mutations if it continues to function after genetic changes in its parts. & [35] \\
\hline & $\begin{array}{l}\text { Biological systems, from macromolecules to whole organisms, are robust if they continue to function, } \\
\text { survive, or reproduce when faced with mutations, environmental change, and internal noise. }\end{array}$ & [21] \\
\hline $\begin{array}{l}\text { Control } \\
\text { theory }\end{array}$ & $\begin{array}{l}\text { A robust system will be able to somehow resist a set of perturbations by providing a graceful loss in } \\
\text { performance. }\end{array}$ & {$[2]$} \\
\hline Engineering & The design [of a robust system] should be safeguarded against uncertain perturbations. & [3] \\
\hline Statistics & $\begin{array}{l}\text { The robustness of a statistical method is related to several classical parametric tests on means and the } \\
\text { population assumptions of normality and equal variances. }\end{array}$ & [5] \\
\hline $\begin{array}{l}\text { Supply } \\
\text { chain } \\
\text { management }\end{array}$ & Robustness refers to the ability of a supply chain to resist or avoid change. & [6] \\
\hline Ecology & Robustness means the capacity of a system to absorb stresses and continue functioning. & [44] \\
\hline \multirow[t]{3}{*}{$\begin{array}{l}\text { Computer } \\
\text { science }\end{array}$} & $\begin{array}{l}\text { Robust computing systems must continue to meet user expectations despite rising levels of } \\
\text { disturbances in the underlying hardware }\end{array}$ & [45] \\
\hline & A robust system should have a high general utility that does any particular job very well. & [4] \\
\hline & $\begin{array}{l}\text { An algorithm is robust if its solution has the following property: it achieves "similar" performance on a } \\
\text { testing sample and a training sample that is "close". }\end{array}$ & [16] \\
\hline Economics & Robustness refers to the insensitivity of the results of inference to alternative specifications. & [46] \\
\hline
\end{tabular}

Table A2. A summary of related concepts on robustness and where they appear in our framework

\begin{tabular}{|c|c|c|c|}
\hline Terms & Description \& Supporting References & $\mathrm{M}^{1}$ & $\mathrm{C}^{2}$ \\
\hline Buffering & $\begin{array}{l}\text { Systems have mechanisms to buffer perturbations; thus, system operations are less affected by } \\
\text { perturbations. [1] }\end{array}$ & $\mathrm{F}$ & $\begin{array}{l}\text { SS\& } \\
\mathrm{R}\end{array}$ \\
\hline Neutral Space & Neutral space is a collection of equivalent solutions to the same biological problem. [29] & $\mathrm{F}$ & R\&D \\
\hline Purging & Purging amplifies the effects of perturbations, to ensure the purity of a population. [28] & I & FL \\
\hline $\begin{array}{l}\text { Spatial } \\
\text { Compartmentaliza } \\
\text { tion }\end{array}$ & $\begin{array}{l}\text { Spatial compartmentalization means a system design that is composed of a finite number of macroscopic } \\
\text { subsystems called compartments, each of which is well mixed. [28] }\end{array}$ & I & $\mathrm{DD}$ \\
\hline $\begin{array}{l}\text { Distributed } \\
\text { Processing }\end{array}$ & $\begin{array}{l}\text { Distributed processing describes those cases in which an integrated set of functions are carried out by } \\
\text { multiple, semiautonomous units. [28] }\end{array}$ & I & $\mathrm{DD}$ \\
\hline $\begin{array}{l}\text { Extended } \\
\text { Phenotypes }\end{array}$ & $\begin{array}{l}\text { Extended phenotype refers to a means of emancipating the gene from the discrete vehicle (often taken to } \\
\text { be the individual organism). [28] }\end{array}$ & $\mathrm{F}$ & SS \\
\hline $\begin{array}{l}\text { Diversity/heteroge } \\
\text { neity }\end{array}$ & $\begin{array}{l}\text { Diversity and heterogeneity capture the adaptive capacity of a system, its ability to alter its composition } \\
\text { in a changing environment. [44] }\end{array}$ & $\mathrm{F}$ & $\mathrm{D}$ \\
\hline Canalization & $\begin{array}{l}\text { Canalization is the extent to which phenotypes remain constant in the face of specified environmental } \\
\text { and/or genetic perturbations. [34] }\end{array}$ & $\mathrm{S}$ & $P$ \\
\hline $\begin{array}{l}\text { Developmental } \\
\text { Control Circuits }\end{array}$ & $\begin{array}{l}\text { Developmental control circuits are related to both operations of individual modules and a rich network } \\
\text { of inter-module communications. [36] }\end{array}$ & I & $\mathrm{DD}$ \\
\hline $\begin{array}{l}\text { Exploratory } \\
\text { Behavior }\end{array}$ & $\begin{array}{l}\text { Exploratory behavior suggests that systems can produce the desired outcome in a generate-and-test } \\
\text { mechanism. [29] }\end{array}$ & $\mathrm{I}$ & FL \\
\hline $\begin{array}{l}\text { Compartments } \\
\text { and Localization }\end{array}$ & Systems are constructed in separate modules. [29] & I & $\mathrm{DD}$ \\
\hline $\begin{array}{l}\text { Defense, Repair, } \\
\text { and Regeneration }\end{array}$ & $\begin{array}{l}\text { Systems have a dynamically reconfigurable structure made out of potentially universal interchangeable } \\
\text { and reproducible parts: if a part is damaged, nearby cells can retarget to fill in the gap and take on the } \\
\text { function of the damaged part. [29] }\end{array}$ & $\mathrm{F}$ & D\&R \\
\hline Composition & $\begin{array}{l}\text { Large systems are composed of many smaller components, each of which contributes to the function of } \\
\text { the whole either by directly providing a part of that function or by cooperating with other components by } \\
\text { being interconnected in some pattern specified by the system architect to establish a required function. } \\
{[29]}\end{array}$ & I & DD \\
\hline $\begin{array}{l}\text { Distributed } \\
\text { Robustness }\end{array}$ & $\begin{array}{l}\text { In distributed robustness, many parts of a system contribute to system function, but all of these parts } \\
\text { have different roles. When one part fails or is changed through mutations, the system can compensate for } \\
\text { this failure, but not because a "back-up" redundant part takes over the failed part's role. [35] }\end{array}$ & $\mathrm{F}$ & $\mathrm{D}$ \\
\hline
\end{tabular}

${ }^{1}[\mathrm{M}]$ - Mechanisms; [F] - Functional; [I] - Infrastructure; [S] -Strategic

2 [C]-Concept; [D] - Degeneracy; [DD] - Distributed Design; [R] - Redundancy; [FL] - Feedback Loop; [P] - Persistence; [SS] - Structural Stability 